\title{
Anomalous Propagation in Low Index Contrast Metamaterials: Assessment of the Beam Collimation Condition
}

\author{
Jun Tan, ${ }^{1}$ Weiwei Song, ${ }^{1}$ and Wei Jiang ${ }^{1,2}$ \\ ${ }^{1}$ Department of Electrical and Computer Engineering, Rutgers University, Piscataway, NJ 08854, USA \\ ${ }^{2}$ Institute for Advanced Materials, Devices, and Nanotechnology, Rutgers University, Piscataway, NJ 08854, USA
}

Correspondence should be addressed to Wei Jiang, wjiangnj@rci.rutgers.edu

Received 1 June 2012; Accepted 10 August 2012

Academic Editor: Georgios Veronis

Copyright (C 2012 Jun Tan et al. This is an open access article distributed under the Creative Commons Attribution License, which permits unrestricted use, distribution, and reproduction in any medium, provided the original work is properly cited.

\begin{abstract}
Anomalous beam propagation in low index-contrast metamaterials has been analyzed. The condition for a well-collimated beam is found to be depending on the beam width and the pertinent Fourier component of the dielectric function. Guided by this condition, an ultra-compact metamaterial structure is designed to deflect a light beam at a wide angle. The structure is tolerant to structural parameter deviation and has a wide bandwidth.
\end{abstract}

\section{Introduction}

Metamaterials have aroused wide spread interest in recent years [1-12]. Many metamaterial structures involve metallic and dielectric components with a large refractive indexcontrast between two material components. Such a large index-contrast results in the capability of confining and maneuvering light path in a very small scale. On the other hand, it is possible to construct metamaterials with relatively low index-contrast. It would be helpful to understand the capability and limit of metamaterials in the low indexcontrast case. In this work, we will study a low indexcontrast metamaterial system composed of polymer and air components in such a context. Negative refraction is a topic of wide interest in metamaterials research [1]. Negative refraction can be considered as one aspect of the anomalous characteristics of an optical beam in metamaterials. We will analyze low index-contrast metamaterials that can produce certain anomalous propagation characteristics. This can be utilized to maneuver light beams of sufficiently large widths with potential applications in polymer photonic integrated circuits [13]. Note that the low index-contrast metamaterials discussed here differ from the low-index (or epsilon-nearzero) metamaterials, whose constitutive components have relatively large indices.
Anomalous beam propagation in metamaterials can be traced back to early studies of photonic crystals in 1998 [14]. Note that photonic crystals can be considered as one category of metamaterials whose refractive indices are usually real. The abnormalities include negative refraction, unusually large refraction/deflection angle, and unusual wavelength sensitivity [14]. The proposed applications of these effects include beam deflectors [15], wavelength-division multiplexers $[14,15]$. To utilize such anomalous propagation in compact devices, it is generally preferred to use a narrow beam at the entrance so that the device lateral width can be minimized. However, the anomalous propagation is usually accompanied by greater angular dispersion, which significantly exacerbates the angular divergence of a narrow beam in a metamaterial. For high index-contrast systems such as silicon-air photonic crystals, this problem can be solved through engineering the dispersion surface [16]. However, this angular divergence problem has not been analyzed in a general manner, especially for metamaterials of small index-contrasts. Note that many low-index materials such as polymers are attractive in a wide range of photonics applications because of their low fabrication/processing cost and flexible form [13]. In this work, we will start from analyzing the beam collimation condition in low index-contrast metamaterials in Section 2. Then Section 3 will present a 
design of a beam deflection device and address some other practical issues of low index-contrast metamaterials. Lastly, conclusions will be presented in Section 4.

\section{Beam Collimation Condition in Low Index-Contrast Metamaterials}

The anomalous propagation characteristics observed in these metamaterials originate from the underlying subwavelength structures. Related problems have been studied in various scenarios in other fields. Particularly, in X-ray diffraction study, a "dynamic theory" has been developed [17] to understand the beam divergence related issue for $\mathrm{X}$-ray propagation in a crystal. Of particular value is the spherical wave part of the dynamic theory of X-ray diffraction. In the limit of zero beam width, the entering beam becomes a completely diverging spherical wave (a cylindrical wave in two dimensional problems), which represents the worst beam divergence scenario. This spherical wave formulation can be easily adapted to help understand the beam divergence issue in a low index-contrast metamaterial. Considering an entering beam with center wavevector $\mathbf{k}_{0}$ diffracted by a Fourier component $\mathcal{E}_{\mathrm{G}}$ of the dielectric function, the first order diffraction has a wave vector $\mathbf{k}_{g}=\mathbf{k}_{0}+\mathbf{G}$, where $\mathbf{G}$ is a reciprocal lattice vector. The dynamic $\mathrm{X}$-ray diffraction theory gives the wave in the crystal as [17]:

$$
\begin{aligned}
E_{g}(x, y)= & A(x, y) \sum_{\sigma= \pm 1} \int_{-\infty}^{+\infty} B(s)\left[\frac{\beta}{\sigma \sqrt{s^{2}+\beta^{2}}}\right] \\
& \times \exp \left[i \sigma \xi_{1} \sqrt{s^{2}+\beta^{2}}-i \xi_{2} s\right] d s
\end{aligned}
$$

where $A(x, y)$ is a fast oscillating field with the ensuing integral being its envelope, $\xi_{1}$ and $\xi_{2}$ represent the local coordinates of an oblique two-dimensional (2D) coordinate system (hence, $\xi_{1}$ and $\xi_{2}$ are two linear functions of $x$ and $y$ ) induced by the dispersion surface; $\sigma=1$ and -1 for two branches of dispersion surface, respectively; $s=\Delta k+C_{1} k_{0}$, with $\Delta k$ being the wavevector deviation from $k_{0} ; \beta=C_{2} k_{0} \varepsilon_{\mathrm{G}}$. The geometric constants $C_{1}, C_{2}$ are of order unity. Here the dispersion surface refers to the constant frequency surface in the reciprocal space. The function $B(s)$ is introduced by us in this work to describe the wavevector distribution (note that $s$ is a function of $\Delta k$ ). The usage of $B(s)$ will be discussed shortly. Equation (1) was obtained through lengthy and complicated derivations, where the physical meaning of the quantities $s, \beta$, and $\xi_{i}$ became obscure. However, it is sufficient to note that the phase factor $\exp \left\lfloor i \sigma \xi_{1} \sqrt{s^{2}+\beta^{2}}-\right.$ $\left.i \xi_{2} s\right\rfloor$ in (1) is equivalent to $\exp \left[i\left(\mathbf{k}-\mathbf{k}_{\mathbf{0}}\right) \cdot \mathbf{r}\right]$, where $\mathbf{k}_{\mathbf{0}}$ is constant. Thus, $s$ and $\sqrt{s^{2}+\beta^{2}}$ are the projections of wavevector deviation $\Delta \mathbf{k}=\mathbf{k}-\mathbf{k}_{\mathbf{c}}$ on the $\xi_{1}$ and $\xi_{2}$ axes, respectively. Note that as $s$ varies, $\beta$ varies accordingly, so that $\mathbf{k}$ remains on the dispersion surface. Therefore, $\beta$ is a function of $s$. In the spherical-wave dynamic X-ray theory [17], due to the limitation of analytic study, only the case $B(s) \equiv 1$ is investigated. It was analytically shown that the wave pattern $E_{g}(x, y)$ spreads within a triangle spanned by $\mathbf{k}_{0}$ and $\mathbf{k}_{g}$ and have hook-shaped interference patterns. For polymer photonic integrated circuits applications, such a triangular spread leads to significant beam divergence, which may increase device lateral dimensions and optical loss. In addition, an additional beam focuser or waveguide taper becomes necessary to couple light into an output waveguide. This increases the device complexity and size.

To overcome these issues originated from beam divergence, it is necessary to quantitatively study the beam divergence with varying structure parameters and seek approaches to minimize the beam divergence under the constraints of small index-contrast. To start, we note that in the X-ray theory, the beam is assumed to originate from a point source with a uniform angular intensity spectrum. Such an ideal simplification is not applicable to integrated optics applications where the input beam has a finite width. We have introduced a wavevector distribution function $B(s)$ in (1) to deal with arbitrary beam profiles. Note that for any given lateral beam profile $b\left(x_{\perp}\right)$ in space $\left(x_{\perp}\right.$ being the coordinate transverse to the beam propagation direction), the corresponding wavevector distribution function $\widetilde{B}\left(\Delta k_{\perp}\right)$ can be obtained from the Fourier transform of $b\left(x_{\perp}\right)$. Note that for a monochromatic beam in 2D space, the wavevector deviation has only one degree of freedom. Therefore, one can easily link $\widetilde{B}\left(\Delta k_{\perp}\right)$ to $B(s)$, noting that $s$ is a linear function of $\Delta k$. Here we address the case where the wavevector is limited to a small angular range such that $\Delta k_{\max } \ll|\beta|$. Assuming a Gaussian beam profile $B(s)$ and expanding (1) in $\Delta s / \beta$ (or equivalently $\Delta k / \beta$ ), one can readily show that for a sufficiently small $\Delta k / \beta \ll 1$, the beam is well collimated without obvious divergence. This case is of our interest here. Introduce the wavevector angle $\theta_{k}$, with respect to the beam axis, we find $\Delta k=k_{0} \sin \theta_{k}$. Also, note $\beta=C_{2} k_{0} \varepsilon_{\mathrm{G}}$, where $C_{2}$ is a constant on the order of unity. Hence, the condition $\left(\Delta k_{\max } \ll|\beta|\right)$ for a well-collimated beam can also be formulated as

$$
\theta_{\mathrm{km}} \ll \varepsilon_{G},
$$

where $\theta_{\mathrm{km}}$ is the characteristic angular spread of the wavevector (roughly speaking, the beam divergence angle) for a finite beam. Consider a Gaussian beam with a width $w$ propagating in a $2 \mathrm{D}$ square lattice of air holes in a medium of relative dielectric constant $\varepsilon_{1}$. It is well known that the beam angular divergence is related to the beam width as $\theta_{\mathrm{km}} \propto \lambda / w$. One can also readily show that the Fourier coefficient $\varepsilon_{G}$ generally has the form of Bessel functions for a photonic crystal composed of circular holes [18]. Thus, based on (2), we can readily show that the condition for a well-collimated beam is given by

$$
w \gg w_{c}=\frac{2 \lambda}{\pi \sqrt{\varepsilon_{1}}\left(\varepsilon_{1}-1\right)} \frac{a}{r J_{1}(2 \pi r / a)},
$$

where $r$ is the hole radius, $a$ is the lattices constant, $\lambda$ is the wavelength, $J_{1}(x)$ is a Bessel function of the first kind, and $w_{c}$ can be regarded a characteristic beam width pertinent to the divergence issue. Assume a metamaterial is composed of air holes in a polymer matrix with typical polymer index $n_{\text {polymer }}=1.5$, we plot $w_{c}$ as a function 


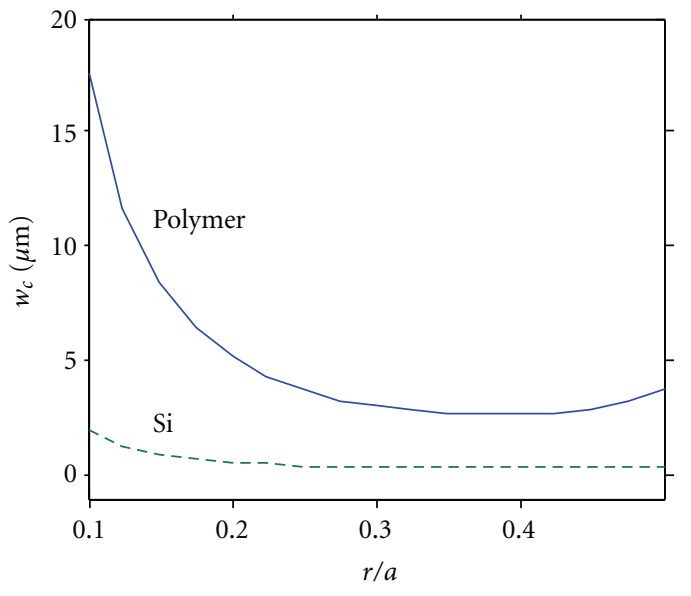

(a)

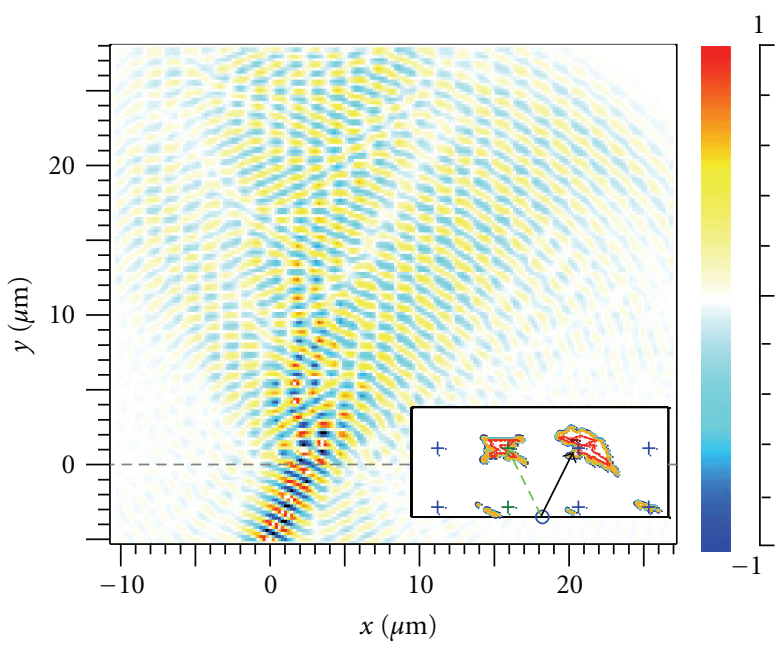

(b)

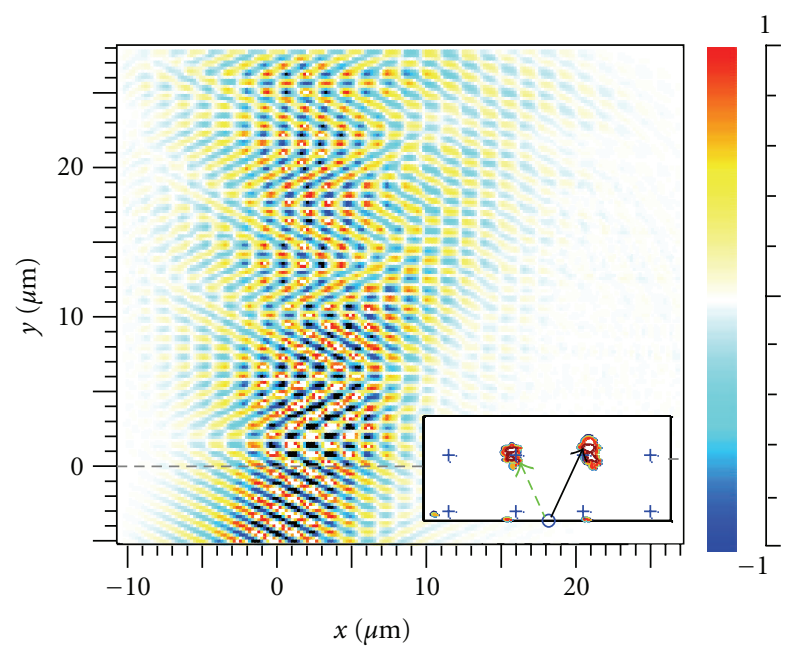

(c)

Figure 1: (a) $w_{c}$ versus $r / a$ curve given by (3) for polymer-based and silicon-based metamaterials. (b) Beam propagation in a polymer metamaterial for a Gaussian beam with a width $w=3 \mu \mathrm{m}$. The metamaterial region starts at $5 \mu \mathrm{m}$ from the bottom of the figure (the interface is indicated by a dashed line) and extends upwards. Inset: 2D Fourier transform of the spatial field in the photonic crystal. The wavevector $\mathbf{k}_{0}$ and $\mathbf{k}_{g}$ are indicated by a black arrow and a green dash arrow. The wavevector of various diffraction orders (on a square lattice in the reciprocal space) are marked by crosses. (c) Same as in (b) except $w=10 \mu \mathrm{m}$.

of $r / a$ in Figure 1(a) for $\lambda=1.55 \mu \mathrm{m}$. For comparison, the $w_{c}$ for a high index-contrast metamaterial composed of air holes in a silicon matrix is also shown. Evidently, the polymer-based metamaterial system requires substantially wider beams than the silicon-based metamaterial system. In this work, the low index-contrast regime pertaining to metamaterial beam collimation refers to $|\Delta n|$ substantially less than unity (e.g., $|\Delta n| \sim 0.5$ ), which is in contrast to high index-contrast metamaterial systems such as silicon-air or silver-air. Note that $w$ should usually be at least 3 to 10 times larger than $w_{c}$ in order to satisfy (3). For the polymerbased metamaterial, the minimal $w_{c} \sim 2.7 \mu \mathrm{m}$ appears at $r / a \sim 0.37$. For $r / a \geq 0.3$, the curve is relatively flat, indicating $w_{c}$ is insensitive to hole radius change in this range. Overall, Figure 1(a) shows that the beam width must be much larger than $3 \mu \mathrm{m}$ for a polymer-based metamaterial at $\lambda=1.55 \mu \mathrm{m}$. Figures $1(\mathrm{~b})$ and $1(\mathrm{c})$ compare two beams of $3 \mu \mathrm{m}$ and $10 \mu \mathrm{m}$ wide, propagating in a $r / a=0.3$ lattice as simulated by the Finite Difference Time-Domain (FDTD) method for polarization normal to the plane. The metamaterial region starts at $5 \mu \mathrm{m}$ from the bottom (each division on the axis is a micron) and extends upward. For the $10 \mu \mathrm{m}$ wide beam case in Figure 1(c), the optical wave inside the metamaterial has the majority of its power propagating in a collimated beam (vertically upwards and slightly tilting to the left), whereas the $3-\mu \mathrm{m}$ wide beam in Figure 1(b) results in a diverging field spreading over a triangle whose left and right edges are roughly parallel to $\mathbf{k}_{\mathbf{0}}$ and $\mathbf{k}_{\mathrm{g}}$. Note that in Figure 1(c), the incident beam points towards the right hand side whereas the beam propagation direction inside the metamaterial slightly tilts towards the left side. This indicates that the beam experiences negative refraction 


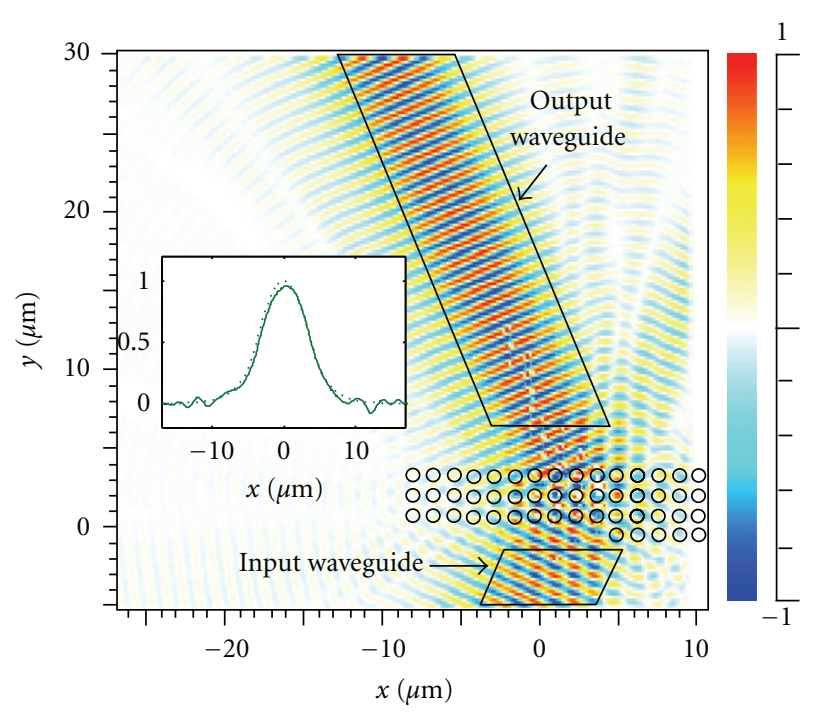

Figure 2: An ultra-compact $47.3^{\circ}$ beam deflector (effectively a wide-angle waveguide bend). Inset indicates the output beam profile (solid curve) is well matched to the waveguide mode profile (dotted line).

at the metamaterial surface, which is anomalous compared to ordinary refraction. For Figure 1(b), the diverging beam wipes out the signature of negative refraction. These FDTD simulation results confirm that the beam width $w$ must be sufficiently larger than the characteristic beam width $w_{c}$ in order to form a well-collimated beam and observe negative refraction in a low index-contrast metamaterial. The insets in Figures 1(b) and 1(c) show the power spectrum of the 2D spatial Fourier transforms of the respective spatial fields. The un-diffracted wavevector $\mathbf{k}_{0}$ is shown by an arrow with the origin $\mathbf{k}=0$ noted by a circle. Because the lower half of the Fourier spectrum is symmetric to the upper half, only the upper half the $2 \mathrm{D}$ Fourier spectrum is shown. The wavevectors of various diffraction orders (on a square lattice in the reciprocal space) are marked by crosses. Their spatial Fourier spectra are dominated by two Fourier components (centered around $\mathbf{k}_{0}$ and $\mathbf{k}_{g}$ ).

\section{Potential Device Application: A Beam Deflector}

A closer look of Figure 1(c) reveals an alternation of two different wave components, whose (center) wavevectors are different from the beam direction. When the beam is collimated as Figure 1(c), this can be utilized to realize lowloss wide angle beam deflection. Figure 2 presents the $2 \mathrm{D}$ FDTD simulation result of a wide-angle $\left(47.3^{\circ}\right)$ deflector, which essentially has the functionality of a wide-angle waveguide bend. The incident beam is a waveguide mode matched to a typical fiber mode, which has a mode width around $10 \mu \mathrm{m}$. The 2D square lattice metamaterial has a lattice constant $a=1.3 \mu \mathrm{m}$, and a hole radius $r=$ $0.3 a$. The lattice constant and hole radius are relatively large to facilitate fabrication. More discussion on practical implementation and fabrication will be presented at the end of this section. High transmission and excellent mode match (see the inset of Figure 2) are achieved at the output end, giving an insertion loss less than $0.5 \mathrm{~dB}$. Note that for the noncollimated case shown in Figure 1(b), because of widely spread beam (whose profile quickly deviates from Gaussian after a short propagation length) inside the metamaterial, it is very difficult to achieve a collimated deflected beam at the output and the loss is usually significantly higher. Note that for the case shown in Figure 2, the beam profile quickly stabilizes after exiting the metamaterial region. The bottom layer of the structure is intentionally designed to be incomplete. Our simulation indicates that such an incomplete layer enhances the transmission. One possible explanation is that when the beam is making a left turn, the right portion of the beam is on the "outer track" and travels a longer distance. Thus, the right portion of the beam also needs a longer interaction length in the metamaterial to complete the turn on the "outer track." Therefore, the right portion of the metamaterial shall be relatively thicker. It is worth noting that at the output surface of the metamaterial region in Figure 2, the beam experiences a second negative refraction. The negative refractions at the front and back surfaces both contribute substantially to producing a large total beam deflection angle.

Figure 3 shows the transmission loss variation with respect to two essential parameters $(\lambda$ and $n)$. For simulation results in Figure 3, the baseline structure has all parameters same as the optimized structure in Figure 2 except that we have used a generic polymer refractive index $n_{\text {polymer }}=1.5$ (the structure in Figure 2 is optimized to have $n_{\text {polymer }}=1.42$ ). This baseline structure has an insertion of $0.7 \mathrm{~dB}$. Additionally, the loss variation with $50 \mathrm{~nm}$ hole radius deviation is less than $0.1 \mathrm{~dB}$. These results show that this device is wide band (whole C-Band) and relatively insensitive to slight deviation of polymer index and fabrication tolerance of the hole radius. Note that a hole radius tolerance of $50 \mathrm{~nm}$ is readily achievable using electron beam lithography. Also, the relative insensitivity to polymer index allows a wide range of polymers to choose from. Note that we have intentionally designed our structure with a relatively large $a$, and an intermediate $r / a$ to facilitate the fabrication. Note the traditional waveguide bends for polymer integrated photonic devices are usually several millimeters long due to the small index-contrast of polymers [13]. The metamaterial structures obviously have advantages in device miniaturization.

For practical implementation of this structure, each waveguide will be a channel waveguide composed of higher index polymer core and lower index polymer cladding, as shown in Figure 4. In the metamaterial region, the core layer will be etched away; thus, the conformal nature of the top polymer cladding will likely lower the top cladding surface to the same height as the top surface of the core layer. To ensure the functionality of the metamaterial, the metamaterial region will be etched to a depth that is greater than the depth the polymer core. Note that polymer waveguides generally have fairly small refractive index-contrasts $(\Delta n \sim 0.01)$ between core and cladding, 


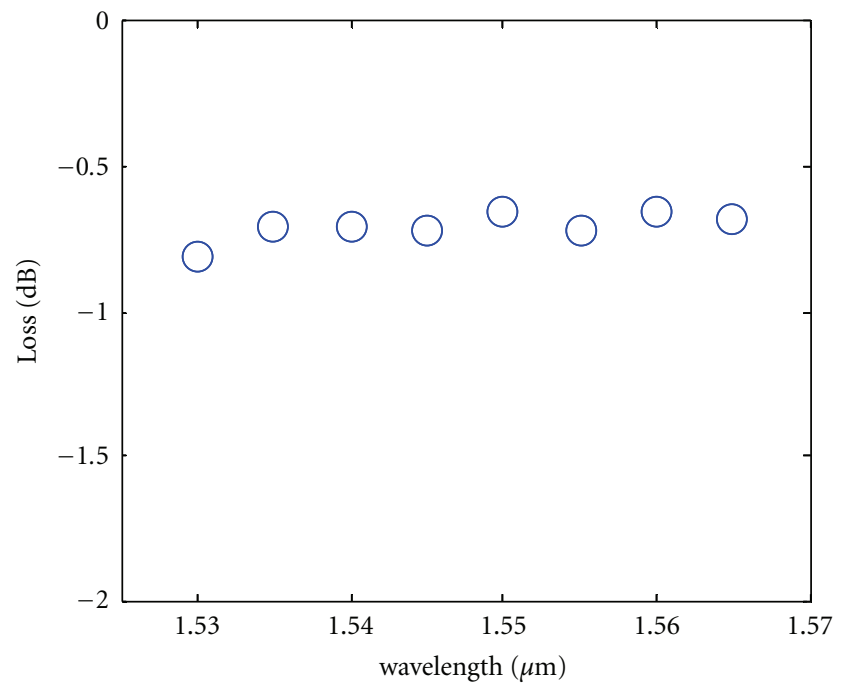

(a)

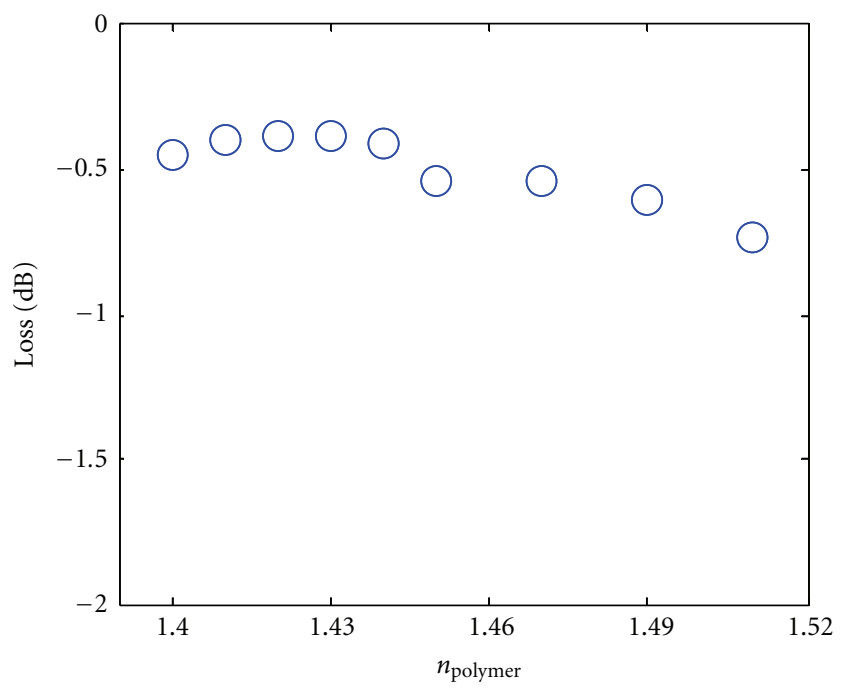

(b)

FIgURe 3: Loss variation with (a) wavelength and (b) polymer index.

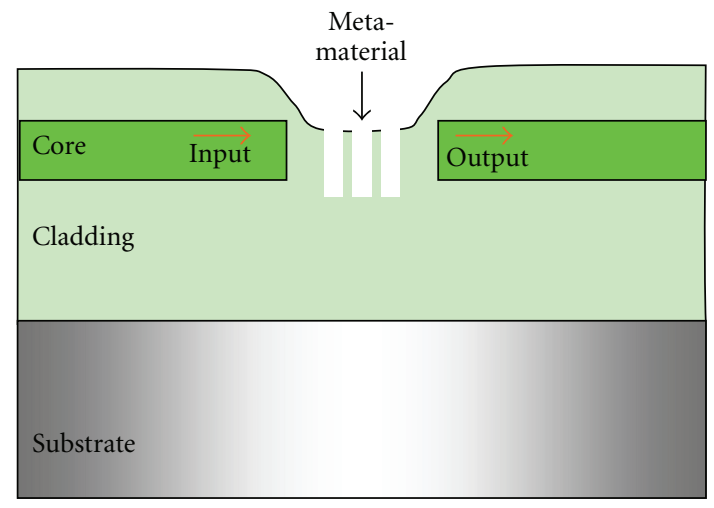

FIGURE 4: Schematic of an example device structure (side view, not drawn to scale).

and relatively large cross-sections. Here we assume a core thickness of $3 \mu \mathrm{m}$ and a top cladding thickness of $2 \mu \mathrm{m}$. As such, it is estimated that we need to etch $\sim 3 \mu \mathrm{m}$ deep into the polymer in the metamaterial region. Although there is no vertical confinement mechanism in the metamaterial region, optical loss due to beam divergence in the vertical direction is expected to be insignificant due to the short length of the metamaterial region ( $\sim \mu \mathrm{m}$ long) through which the beam travels.

\section{Conclusions}

In summary, the condition for achieving well-collimated beams in a metamaterial has been analyzed. Guided by this analysis, we have designed ultra-compact, wide-angle beam deflection devices that are significantly smaller than conventional polymer integrated photonic devices. The simulations also show that these devices have a wide bandwidth and are insensitive to fabrication tolerances. A wide range of polymers can be chosen to fabricate these structures. Such polymer photonic devices are attractive for their compact size, low cost and flexible form.

\section{Acknowledgment}

This work is supported in part by AFOSR Grant No. FA955008-1-0394.

\section{References}

[1] D. R. Smith, J. B. Pendry, and M. C. K. Wiltshire, "Metamaterials and negative refractive index," Science, vol. 305, no. 5685, pp. 788-792, 2004.

[2] V. M. Shalaev, W. Cai, U. K. Chettiar et al., "Negative index of refraction in optical metamaterials," Optics Letters, vol. 30, no. 24, pp. 3356-3358, 2005.

[3] S. Zhang, W. Fan, N. C. Panoiu, K. J. Malloy, R. M. Osgood, and S. R. J. Brueck, "Experimental demonstration of near-infrared negative-index metamaterials," Physical Review Letters, vol. 95, no. 13, Article ID 137404, 4 pages, 2005.

[4] S. Linden, C. Enkrich, M. Wegener, J. Zhou, T. Koschny, and C. M. Soukoulis, "Magnetic response of metamaterials at 100 terahertz," Science, vol. 306, no. 5700, pp. 1351-1353, 2004.

[5] D. Schurig, J. J. Mock, B. J. Justice et al., "Metamaterial electromagnetic cloak at microwave frequencies," Science, vol. 314, no. 5801, pp. 977-980, 2006.

[6] H. J. Lezec, J. A. Dionne, and H. A. Atwater, "Negative refraction at visible frequencies," Science, vol. 316, no. 5823, pp. 430-432, 2007.

[7] N. Engheta, "Circuits with light at nanoscales: optical nanocircuits inspired by metamaterials," Science, vol. 317 , no. 5845, pp. 1698-1702, 2007.

[8] J. A. Schuller, R. Zia, T. Taubner, and M. L. Brongersma, "Dielectric metamaterials based on electric and magnetic resonances of silicon carbide particles," Physical Review Letters, vol. 99, no. 10, Article ID 107401, 4 pages, 2007. 
[9] J. Valentine, S. Zhang, T. Zentgraf et al., “Three-dimensional optical metamaterial with a negative refractive index," Nature, vol. 455, no. 7211, pp. 376-379, 2008.

[10] S. E. Kocabaş, G. Veronis, D. A. B. Miller, and S. H. Fan, "Transmission line and equivalent circuit models for plasmonic waveguide components," IEEE Journal on Selected Topics in Quantum Electronics, vol. 14, no. 6, pp. 1462-1472, 2008.

[11] W. Zhao, X. Huang, and Z. Lu, "Super Talbot effect in indefinite metamaterial," Optics Express, vol. 19, no. 16, pp. 1529715303, 2011.

[12] J. X. Chen, P. Wang, C. C. Chen, Y. H. Lu, H. Ming, and Q. W. Zhan, "Plasmonic EIT-like switching in bright-dark-bright plasmon resonators," Optics Express, vol. 19, no. 7, pp. 59705978, 2011.

[13] R. T. Chen, "Polymer-based photonic integrated circuits," Optics and Laser Technology, vol. 25, no. 6, pp. 347-365, 1993.

[14] H. Kosaka, T. Kawashima, A. Tomita et al., "Superprism phenomena in photonic crystals," Physical Review B, vol. 58, no. 16, pp. 10096-10099, 1998.

[15] T. Baba and M. Nakamura, "Photonic crystal light deflection devices using the superprism effect," IEEE Journal of Quantum Electronics, vol. 38, no. 7, pp. 909-914, 2002.

[16] B. Momeni, J. Huang, M. Soltani et al., "Compact wavelength demultiplexing using focusing negative index photonic crystal superprisms," Optics Express, vol. 14, no. 6, pp. 2413-2422, 2006.

[17] L. V. Azaroff, R. Kaplow, N. Kato, R. J. Weiss, A. J. C. Wilson, and R. A. Young, X-Ray Diffraction, McGraw-Hill, New York, NY, USA, 1974.

[18] W. Jiang and R. T. Chen, "Symmetry-induced singularities of the dispersion surface curvature and high sensitivities of a photonic crystal," Physical Review B, vol. 77, no. 7, Article ID 075104, 8 pages, 2008. 

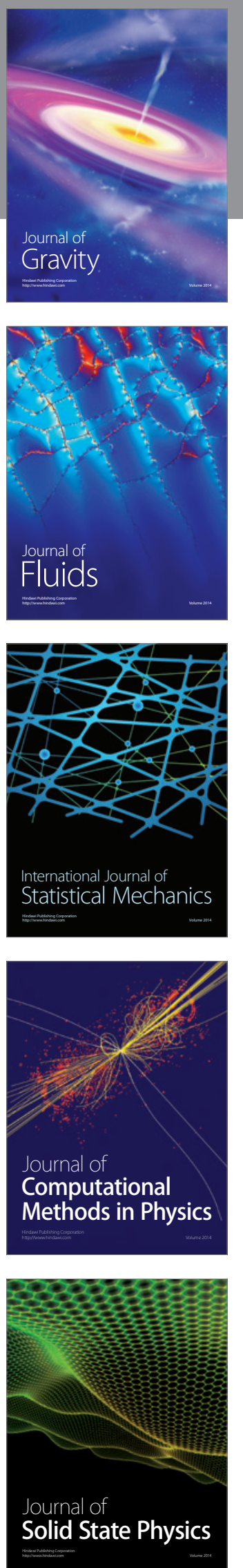

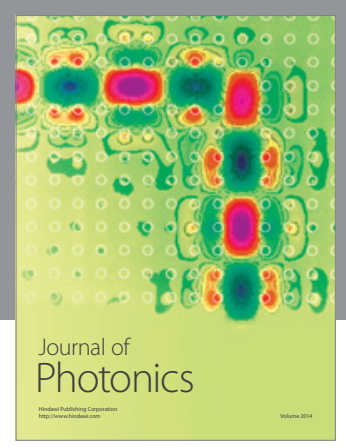

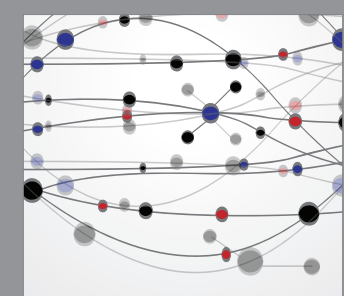

The Scientific World Journal
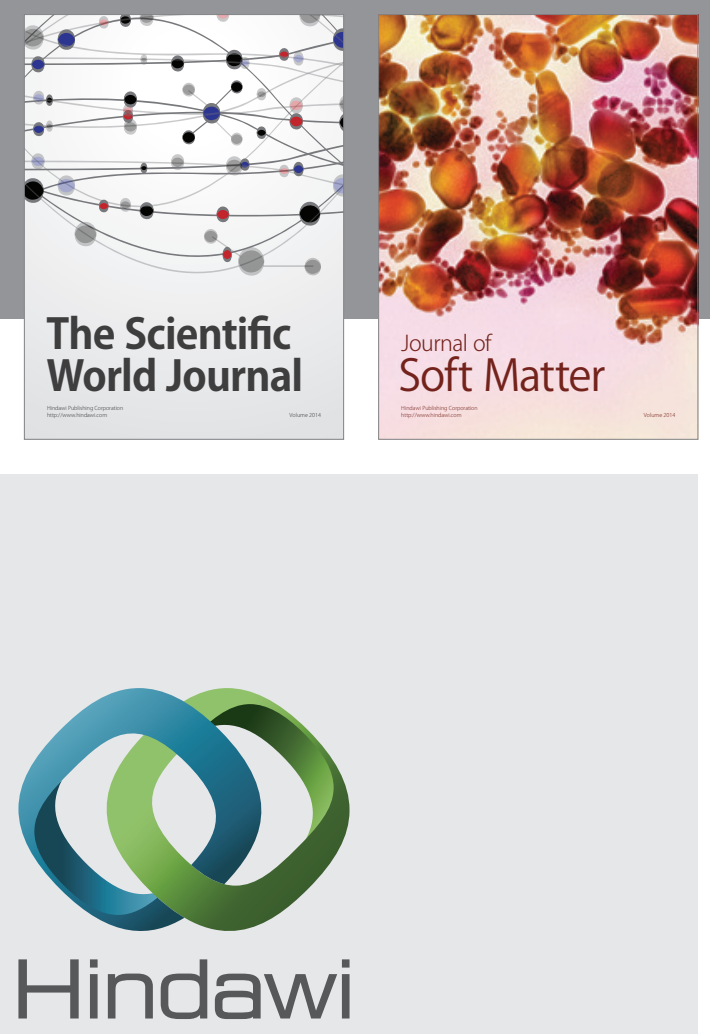

Submit your manuscripts at

http://www.hindawi.com
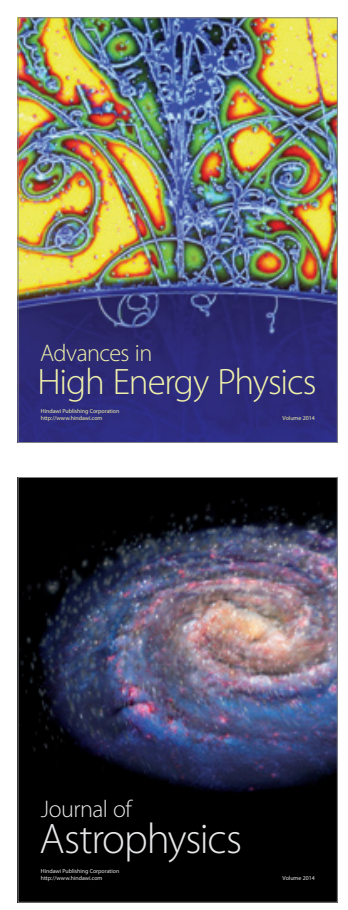
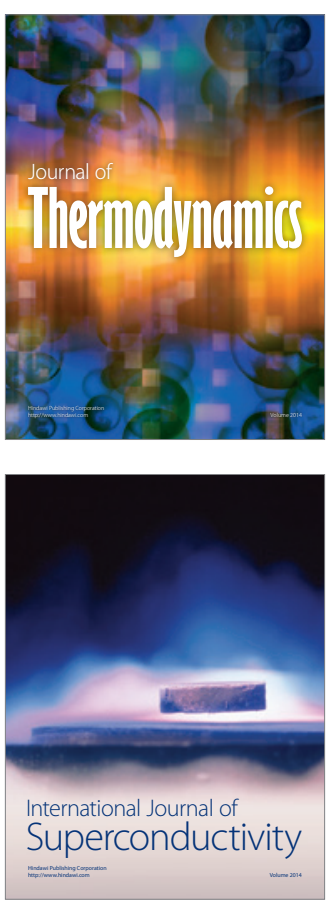
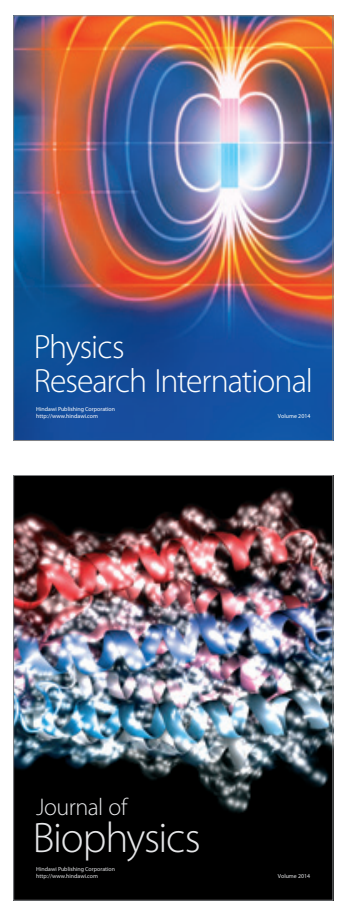
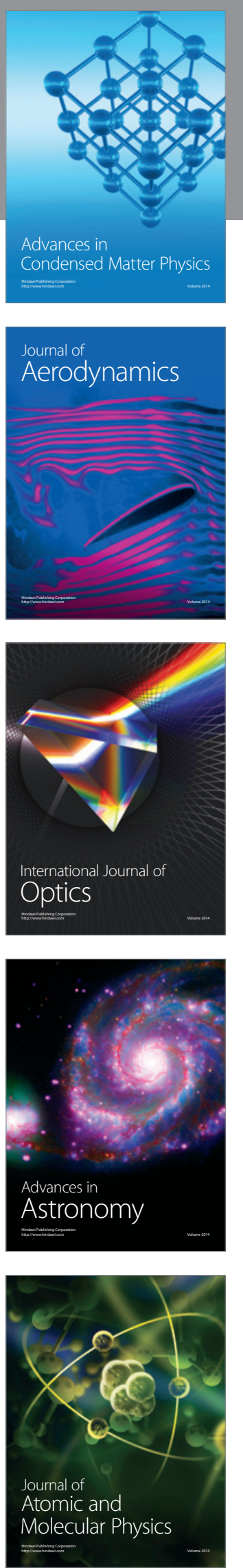